Spring 5-1-2015

\title{
Caregiving factors affecting breastfeeding duration within a neonatal intensive care unit.
}

\author{
Sharon G. Casavant \\ University of Connecticut School of Nursing, sharon.casavant@uconn.edu \\ Georgine Burke \\ University of Connecticut School of Medicine and Connecticut Children's Medical Center, Gburke@connecticutchildrens.org \\ Carrie-Ellen Briere \\ University of Connecticut School of Nursing, Connecticut Children's Medical Center, cbriere@connecticutchildrens.org \\ Jacqueline McGrath \\ University of Connecticut School of Nursing, jacqueline.mcgrath@uconn.edu
}

Follow this and additional works at: https://opencommons.uconn.edu/usp_projects

Part of the Critical Care Nursing Commons, and the Maternal, Child Health and Neonatal Nursing Commons

\section{Recommended Citation}

Casavant, Sharon G.; Burke, Georgine; Briere, Carrie-Ellen; and McGrath, Jacqueline, "Caregiving factors affecting breastfeeding duration within a neonatal intensive care unit." (2015). University Scholar Projects. 18.

https://opencommons.uconn.edu/usp_projects/18 
Caregiving factors affecting breastfeeding duration within a neonatal intensive care unit.

Sharon G. Casavant, University Scholar, BSN Candidate University of Connecticut, School of Nursing, Storrs, CT, USA. 231 Glenbrook Road, U-2026, Storrs, CT, 06269-2026.

Telephone: 860.884.0626, Email: Sharon.casavant@uconn.edu (Corresponding author)

Georgine Burke, Ph.D.

Connecticut Children's Medical Center, 282 Washington Street Hartford, CT, 06106, USA. University of Connecticut School of Medicine, Farmington, CT, USA

Carrie-Ellen Briere, $\mathrm{PhD}, \mathrm{RN}$ Connecticut Children's Medical Center, 282 Washington Street Hartford, CT, 06106, USA. University of Connecticut School of Nursing, Storrs, CT, USA

Jacqueline McGrath, PhD, RN, FNAP, FAAN University of Connecticut School of Nursing, 
231 Glenbrook Road, U-2026, Storrs, CT, 06269-2026.

Telephone: 860.486.4435. Email: $\underline{\text { Jacqueline.mcgrath@uconn.edu }}$

Connecticut Children's Medical Center, Hartford, CT, USA.

Funding provided by the University of Connecticut, Office of Undergraduate Research, Summer Undergraduate Research Fund.

Casavant, S.G., McGrath, J.M., Burke, G., Briere, C. (in production). Caregiving factors affecting breastfeeding outcomes of very low birthweight premature infants within the NICU. Advances in Neonatal Care. 


\section{Abstract}

Background: Increasingly, evidence supports oral feeding of very-lowbirth-weight (VLBW) preterm infants exclusively at breast or with breastmilk. Despite known breastmilk benefits, outcomes related to exclusive breastmilk provision are poor. Identifying factors that promote breastmilk provision is critical.

Purpose: Breastfeeding practices of mothers of VLBW infants admitted to neonatal intensive care unit (NICU) were explored to identify factors associated with mode of feeding at discharge.

\section{Method:}

This retrospective study replicates previous work. Subjects were VLBW preterm infants consecutively admitted during a 24-month period. Primary outcomes included receiving any breastmilk at discharge. Infant variables included gestational age (GA), post-menstrual age (PMA) of first direct breastfeeding, and co-morbid conditions. Maternal variables included age and ethnicity. Nursing practices variables included first direct-to-breast feeding, number of times to breast daily and total direct-to-breast feeding encounters 24 hours prior to discharge. 
Results: 96 VLBW infants $(28.7 \pm 2.8$ weeks GA) met inclusion criteria. Of these, $48 \%$ received breastmilk at discharge. Controlling for significant effect of length of stay, infants receiving first oral feed at breast were more likely discharged home receiving breastmilk (adj OR 8.7, 95\% Cl 2.9-32.3, $p<0.0001)$. There was both an independent effect of first oral feed at breast, and an interaction where infants of non-married women also benefited from the first oral feed at breast.

Implications: Significant associations were found between first oral feeding at breast and infant receiving any breastmilk at discharge. Targeting VLBW infants to receive first oral feeding at breast may yield the best outcome even among sickest and smallest infants.

Keywords: breastmilk, breastfeeding, very low birthweight preterm infants, premature infants, exclusive breastfeeding

\section{What this Study adds:}

- First oral feed being directly at breast increases the odds of continuing to provide some breastmilk through discharge; 
- Provision of multiple (more than one) direct-to-breast feeds per day also positively correlated to some continued breastmilk provision through discharge.

\section{Introduction}

As technology advances, greater numbers of very low birth weight (VLBW) preterm infants are surviving. At the same time, research supporting breastmilk feeding has gained momentum. The benefits to feeding VLBW preterm infants exclusively with breastmilk may be improved when the feedings are provided directly at the breast or with lengthier provision of breastmilk via breast or bottle. Among others, benefits include decreased rates of necrotizing enterocolitis and fewer days requiring total parenteral nutrition. ${ }^{1}$ Evidence indicates that VLBW preterm infants who received mother's own milk experienced reduced incidence of late-onset sepsis, necrotizing enterocolitis, chronic lung disease, retinopathy of prematurity, decreased lengths of NICU stays and reduced rehospitalization rates. ${ }^{2-9}$ These benefits are related to the natural immunity breastmilk confers, as well as improved myelination of neurons via docosahexaenoic acid(DHA) and the improved mother-infant bonding via oxytocin. ${ }^{2-9,24,25}$ These are critical to improving the health outcomes and quality of life for these very fragile infants. These findings are the basis for the World Health Organization in their 2011 statement which 
calls for exclusive breastmilk feedings for all infants in at least the first six months of life to achieve optimal growth, development, and health. ${ }^{10}$ For VLBW preterm infant-mother dyads, this can be particularly challenging since it may be weeks or even months before the infant is able to tolerate oral feeds. Instead, these infants often receive gastric feedings until such time as they are able to coordinate the suck/swallow/breathe action required for oral feedings. An unfortunate result of this late introduction at the breast is that in many cases the mother's milk supply has declined during the several weeks of breast pumping. ${ }^{11}$ In addition to the delay in infant's readiness to feed, caregiving practices and/or experiences in the NICU may also affect initiation of direct-to-breast-feeding, thus identifying those practices is critical. ${ }^{12}$ This vulnerable population stands to significantly benefit from the conferred immunity and easily digestible nutrients found within breastmilk. Thus, the purpose of this study was to explore maternal breastfeeding practices of VLBW preterm infants $(\leq 1500$ grams) admitted to the neonatal intensive care unit (NICU). Specifically, we intended to identify nursing and parental caregiving factors associated with mode of feeding at discharge.

This study used a retrospective descriptive design and is a replication of one conducted by Pineda. ${ }^{13}$ Pineda's study used a retrospective cohort of 84 VLBW infants discharged from a Level II and III 
NICU in a mid-sized southern city. Our sample size was larger $(n=96)$, and took place in a level IV NICU in an urban New England hospital where excellent breastfeeding support existed. Pineda examined associations between breastmilk feedings at NICU discharge and whether an infant was ever put directly at breast in the NICU, number of times the infant was put directly to breast, whether the first oral feed was directly at breast, and, gestational age at first direct-to-breast feed (for both studies, breast milk provision is defined as delivery of nutrients in breastmilk whether by bottle, directly-at-breast or a combination). Although it is a challenge for mothers to pump breastmilk until such time as the infant is able to orally feed, ${ }^{14}$ once direct breastfeeding begins, mothers are better able to continue providing breastmilk through discharge. ${ }^{13}$ One of the key findings of Pineda's study was that of the mothers who were able to provide their infants' first oral feed at breast, $60 \%$ were still providing breastmilk upon discharge. In contrast, of the mothers whose infants did not receive their first oral feed at breast, only $30 \%$ were still providing breastmilk upon discharge indicating a potential association between first oral feed being provided directly at breast and the ability to continue providing breastmilk until discharged home. Pineda's findings did not reach statistical significance, prompting her to suggest that a larger sample size might provide enough statistical power to validate this finding. We found these 
findings striking. These interesting findings provide the basis and rationale for our replication study. Using the results of Pineda's study, we questioned whether our results would be at least similar if not significantly better given our larger sample size and increased resources for supporting lactating mothers in the NICU. Thus, our specific aims and research questions were:

Specific Aim 1: Examine recent practices of mother's who breastfed their VLBW preterm infants in the NICU.

Research question 1: Will at least $90 \%$ of mothers provide some breastmilk in the first week of life?

Research question 2: Will at least $30 \%$ of mothers who initiated breastmilk feedings in the first week of life will still be providing some breastmilk at discharge from the NICU?

Specific Aim 2: Identify nursing and parental caregiving factors that may encourage or inhibit continued provision of breastmilk or breastfeeding.

Research question 3: Will incidence of continued breastmilk provision at discharge positively correlate with first oral feeding at breast (direct-to-breast feeding)? 
Research question 4: Will incidence of continued breastmilk provision at discharge positively correlate with receipt of any direct breastfeeding?

\section{Methods}

\section{Study Design and Setting}

This retrospective chart review replicates previous work and was approved by the Institutional Review Boards (IRB) of a university and the subject hospital. The benefit of conducting an independent replication study is that it allows us to "verify an empirical generalization" by introducing modifications. ${ }^{15}$ Pineda indicates that a larger sample size might further elucidate the trending significance she found, thus, we used a larger sample size of 96 VLBW infants. While replication studies are often cast aside in favor of original work, replication provides necessary feedback among scientists. In 1975, Finifter elaborated saying:

"The fundamental process of scientific communication feedback consisting of continual dialogues among researchers over the production of knowledge whose authenticity and value depend on empirical demonstrations of (a) its reproducibility; (b) its validity; (c) its generalizability over a specified range of instances and contexts; and (d) its extendibility by logically consistent inferences to additional consequences (p. 122)."16 
Data collection was conducted at a level IV 32-bed urban NICU in New England. The study setting is relatively unique in that there are three Internationally Board Certified Lactation Consultants providing clinical support related to milk expression and breastfeeding from 8 a.m. to 8p.m., seven days per week. In addition, almost a third of the staff nurses working in this NICU also hold certification as lactation consultants. Sample Selection

Preterm infants were included if they were consecutive new admissions born during a 24-month period (May 1, 2011 - May 1, 2013) with a birth weight equal to or less than $1500 \mathrm{~g}$. See Table 1 for exclusion criteria.

--Insert Table 1. Exclusion Criteria--

\section{Procedures}

Informed consent was waived given this was a retrospective review and no identifying information were being collected. At the study site, volume and mode of infant feeding were documented within each infant's chart by nursing staff. Those who were bottle-fed (formula or breastmilk) simply indicated the number of milliliters consumed while infants who fed directly-at-breast were weighed prior to feeding and immediately after feeding to determine the amount consumed. A retrospective chart review 
was conducted by the first author. In addition, a second investigator, blinded to the research questions, reviewed each chart. Inter-rater reliability was $96 \%$ agreement. From the charted documentation, infant characteristics included whether the infant was ever put directly to breast, gestational age at the first feeding at breast, number of times the infant was put directly to breast, and whether the infant received breast milk exclusively within the last 24 hours prior to discharge. The primary outcome was percent of infants receiving breastmilk within the 24 hours prior to NICU discharge, either exclusively from the breast or a combination of direct breastfeeding and breastmilk by bottle.

Statistical analysis was conducted using JMP, version 11, SAS Institute, Cary, NC. Descriptive statistics were calculated for all measures. The association of breast milk feeding at discharge with number of breast milk feedings per day and maternal, infant and environmental factors were evaluated with statistical tests appropriate for level of measurement, including Kruskal-Wallis, one-way non-parametric analysis of variance, student $\mathrm{t}$ test or chi square. Logistic regression models with any breastmilk at discharge as the dependent variable were evaluated, and dichotomized (median) variables retained in the model according to the percent change in the model log likelihood ratio. The final model contained only predictors with the probability of error $<0.05$ and a whole model $p<0.05$. Finally, we 
dealt with redundancy in the maternal variables resulting from the multiple gestations by randomly eliminating one delivery from the maternal data (or two in the one case of triplets) and testing the regression models separately. In all cases, the effect sizes for predictor variables were the same. The results shown are for all 96 infants.

\section{Results}

The final study cohort consisted of 96 VLBW preterm infants and 87 mothers. Please see Figure 1 for additional cohort information. Of the 96 infants discharged directly from the study site during the 24-month data collection period, 95 received at least one feeding of their mother's own milk (99\%) during the first week of life, thus supporting research question $\# 1$, and 60 of those went directly to breast for at least one feed (66\%) when developmentally able. Forty-six infants (48\%) were discharged receiving some breast milk whether direct-to-breast or breastmilk via bottle, thus supporting research question \#2. In addition, the sample size of $96 \mathrm{had}>90 \%$ power ( 0.05 significance level) to detect a difference between first oral feed being direct-to-breast and receiving breastmilk at discharge.

-- Insert Figure 1. Diagram of Infant Participants and Outcome -- 
The range of gestational age at birth was 24 weeks to 37 weeks, (28.8 \pm 2.9 weeks). Birth weights ranged from 484 to 1490 grams, (1024 \pm 268 grams). Please see Table 2. for more details of study infants. Among mothers, $63 \%$ of the sample $(n=55)$ were Caucasian (includes those of Hispanic ethnicity), 26\% ( $n=23)$ were African-American. One quarter $(n=22)$ identified as Hispanic. The majority were married (self report) $(n=51) 59 \%$. The average age at delivery was $28.5 \pm 6$ years.

-- Insert Table 2. Infant Characteristics and Table 3. Comparison of Infants Who Were or Were Not Receiving Breastmilk at Discharge--

Thirty-eight infants (40\%) had their first oral feed direct-to-breast. First oral feedings direct-to-breast took place between 31 and 37 weeks PMA ( $M=33.9 \pm 1.0$ weeks). Fifty-nine percent of mothers who were able to provide their infant's first oral feed direct-to-breast were discharged still providing any breastmilk (27 of 46 mothers) thus, supporting research question \#3. Forty-two infants in the study sample received multiple oral feeds at breast daily. Of these 34 were discharged receiving at least some breastmilk. Among the 19 mothers whose infants did not receive their first oral feed direct-to-breast but were discharged providing at least some breastmilk, 10 initiated multiple direct-to-breast feedings per day (mean 
number of direct-to-breast feedings during the entire NICU stay and s.d. were $15 \pm 22$ ). Of those 10 mothers providing multiple direct-to-breast feeds per day, 8 were discharged still providing breastmilk to their infants thus, supporting our research question \#4 about the importance of supporting multiple breastfeeding opportunities during the NICU stay.

-- Insert Table 3. Comparison of Infants Who Were or Were Not Receiving Breastmilk at Discharge -

Table 3. provides the associations among study variables with the main study outcome (infants receiving at least some breastmilk at NICU discharge). Mothers who were still providing some breastmilk at discharge were older and more likely to be married. There was no direct relationship with ethnicity. They were less likely to have delivered vaginally. Infants in this group were somewhat larger at birth, though they did not differ in gestational age at first oral feed from infants who were not discharged receiving breastmilk. They were less likely to have needed surfactant and were discharged, on average, 22 days earlier. $p<0.0001$.

We found a significant relationship between whether the first oral feeding was directly at breast and the likelihood the infant was still receiving any breastmilk at discharge $(p=<0.0002)$. Other caregiving 
factors associated with breastmilk feeding at discharge (not shown in table) included whether the infant was ever directly breast-fed during the NICU stay $(p=<0.001)$ and the number of times the infant was put directly to breast $(p=<0.003)$.

Multiple logistic regression identified the independent contribution of infant and maternal characteristics to the outcome of any breastmilk at discharge. In order to avoid problems of multicolinearity, each of the caregiving factors associated with breast milk feeding were evaluated separately and the one with the largest impact (log likelihood chi square) was retained. As shown in Table 4, length of stay $<60$ days (median) and first oral feed to breast were independently associated with the outcome, with the odds of breastfeeding at discharge at 4.65 and 8.7, respectively. Mother's marital status was not associated independently; rather, the influence of first oral feed at breast was significant for unmarried mothers only. Maternal age, infant comorbid conditions, birthweight and other maternal characteristics were not associated with outcome.

The primary findings of this study indicate that a VLBW premature infant's odds of being discharged still receiving breastmilk correlate to a) first oral sucking feed being directly-at-breast; and, b) the mother putting 
the infant directly-to-breast multiple times per day. While it is not possible to know the exact reason behind the correlation of first oral sucking feed at breast and likelihood of providing breastmilk through discharge, we can infer that it is a combination of maternal feelings of self-efficacy, being able to provide for her infant and the unique sense of well-being and bonding that occurs between mother and child through the neuroendocrine release of oxytocin. ${ }^{24}$

Receiving multiple direct-to-breast feeds per day was also correlated with receiving some breastmilk at discharge. It is important to note that most of those infants who received multiple direct-to-breast feeds per day and were discharged receiving some breastmilk, were on average larger at birth and more likely to be delivered vaginally as compared to infants not receiving any breastmilk at discharge. Additionally, these larger infants were less likely to have received surfactant for risk of respiratory distress syndrome (RDS) and had shorter lengths of stay in the NICU (see Table 3.). While we note that these infants were more healthy and/or larger in size, all infants included in this study were $\leq 1500 \mathrm{~g}$ at birth (see Table 1 ).

Interestingly, data analysis showed that un-married mothers who provided their infant's first oral feed directly at breast were 12 times more likely to be discharged still providing breastmilk via direct breastfeeding or 
bottle. These mothers are more likely to have had less prenatal care, be younger, lower belief in self-efficacy and have less breastfeeding education. ${ }^{26-28}$ Our findings may be a result of the significant lactation support provided at this site.

Of the $48 \%$ of mothers who continued to provide breastmilk until discharge, $100 \%$ of these mothers provided at least one direct-to-breast feeding in the 24 hours prior to discharge. It is possible that the rationale for our results relates to the uniqueness of the setting and the amount of lactation support provided to NICU mothers. We believe these finding provide important support for increasing the degree of lactation support in the NICU.

In Pineda's study (2011) 16.4\% ( $n=11)$ of mothers who initiated breast milk feedings in the NICU had their first oral feeding directly at the breast. Our findings substantiate Pineda's trend that first-oral-feed at breast correlates with the infant continuing to receive breastmilk upon discharge. We found that infants who received their first oral feeding directly at breast were 8 times more likely to continue to receive breastmilk feedings until discharge from the NICU. Gestational age was not a significant factor in our analysis. These findings suggest that targeting VLBW infants to receive their first oral feeding at breast may yield a significant benefit, even among the smallest and sickest infants. While 
cue-based feeding has become the feeding method of choice in many NICUs ${ }^{17}$, waiting or facilitating the mother to be present for the provision of the first oral feeding may be more important to overall long term infant outcomes. Finding ways to best support mother's presence during this transition needs to be a focus of our caregiving.

A recent nurse-based initiative focusing on improving provision of direct-at-breast feedings at the Winnie Palmer Hospital for Women \& Babies in Orlando, Florida has had significant success. ${ }^{24}$ This initiative encouraged NICU mothers to continue breast pumping until babies were able to orally feed. The mothers were asked if they wanted their baby's first oral feed to be at breast and if so, a sign was placed on the crib to indicate this desire and also served as a reminder to both mother and staff. Nurses who promoted mothers to achieve the first oral feed at breast were recognized on a "celebration board". Six months after the initiative began, $92 \%$ of mothers were providing their first oral feed at breast and, of those participants, $92 \%$ of infants were discharged receiving maternal breastmilk. ${ }^{24}$ Integrating this initiative into a policy change and thus, provides support for mothers to offer the first oral feeding directly at breast would be a next step in making this caregiving practice a routine behavior in the NICU. More research around this issue is needed to better understand the risks and benefits of these caregiving practices. 
Recently, Maastrup et al's team had similar findings. Their study found that $95 \%$ of preterm infants who were discharged exclusively breastfeeding received their first oral feed directly from the breast. ${ }^{18}$ These findings, combined with ours and others who have investigated the challenges of breastmilk provision support implementing breastfeeding activities and protocols within the $\mathrm{NICU}^{-19}$. However, it is important to note that the Maastrup's study was conducted in Sweden where family centered care and breastfeeding practices are integrated differently than in the United States. Initiatives such as the Baby Friendly initiative are paving the way for more support of mothers who are providing breastmilk or breastfeeding. Even more important, decreasing mother-infant separation has implications for more positive infant development on many fronts, including increased milk production, better maternal identity, decreased maternal anxiety and depression as well as better infant neurodevelopment ${ }^{18}$. How we facilitate "family" has implications both in the NICU and after discharge. Continuity of breastfeeding must be an aspect of the transition from the NICU to the community and must be facilitated by both NICU providers and those who will follow the family after discharge.

Our second finding indicates that even if a mother is unable to provide her infant's first oral feed directly-at-breast, if she is able to 
provide multiple direct-to-breast feeds per day, there is greater likelihood that she will still be providing some breastmilk at discharge. However, as noted earlier, this finding was more likely to occur with larger, healthier infants who had shorter lengths of stay.

Additionally, Pineda's study identified some of the barriers to breastmilk feeding of VLBW infants in the NICU, including diminished milk supply during the long weeks and months before these infants are able to be fed orally. There are many reasons for diminished milk supply, we only address a few, such as the fact that while breast pumps are necessary, they may impede milk production ${ }^{20-22}$. For example, a breast pump cannot stimulate a mother's endocrine response in the same manner as a suckling baby. Infant suckling yields a surge of prolactin and oxytocin. Prolactin aids in milk production while oxytocin aids in milk ejection and hormonally stimulates bonding between mother and infant. ${ }^{20-22}$ In addition, there is evidence that these processes also program the body for longterm milk production. ${ }^{20-22}$ Thus, pumping for several weeks as opposed to a shorter duration of time decrease the odds that these vulnerable infants will reap the benefits of direct-to-breast feeding. Facilitating skin-to-skin contact has been found to increase breastmilk production and can be an opportunity for first suckling at the breast to occur ${ }^{-23}$. 
As with most research, the benefit of this study is that it allowed identification of factors requiring additional investigation. Such factors include both the physiologic as well as the psychological factors affecting mother's ability to provide direct-to-breast feeding of their VLBW infants and why some mothers who initiated direct-to-breast feeding did not provide multiple direct-to-breast feedings per day.

\section{Limitations}

While these findings are important in broadening our knowledgebase, they are not without limitations. The study was conducted in only one setting, and that setting happens to be one where breastfeeding practices are well-supported. Given that this study was a retrospective chart review, there are some missing data as well as some variables needing to be inferred such as using surfactant as a proxy for RDS risk co-morbidities including length of ventilation and use of CPAP and thus, acuity of illness. In addition, since we used weight as a cut-off for study inclusion (as was done in the previous study) we did not consider some additional variables that could affect the results such as excluding infants who were less than or equal to 32 weeks since those that are older could be small for gestational age or had intrauterine growth restriction. Other variables not considered were provision of skin-to-skin contact, nonnutritive breastfeeding (i.e. suckling which is not considered a feeding), 
breastfeeding experience, distance from the hospital, etc. An

observational prospective study might yield more comprehensive findings.

$$
\text { -- Insert Summary Table Here -- }
$$

\section{Conclusion}

While mother's whose very low birthweight preterm infants are admitted to a NICU face a host of challenges, providing breastmilk through discharge may not need to be one. Our evidence suggests that first oral feed being direct-to-breast increases the odds of being discharged still receiving some breastmilk. Thus, encouraging NICU staff to promote first oral feed being directly at-breast may have far-reaching effects for these high-risk infants and their families, improving physiological and mental health outcomes far into the future.

\section{References}

1. Ghandehari $\mathrm{H}$, Lee $\mathrm{M}$, Rechtman D. An exclusive human milk based diet in extremely premature infants reduces the probability of remaining on total parenteral nutrition: a reanalysis of the data. BMC Res Notes. 2012,5:188. Doi:10.1186/17560500-5-188. 
2. Patel AL, Meier PP, Engstrom JL. The evidence for use of human milk in very low-birthweight preterm infants.

NeoReviews. 2007; 8(11): e459-e466.

3. Meier PP, Engstrom JL, Patel AL, Jegier BJ, Bruns N. Improving the use of human milk during and after the NICU stay. Clin Perinatol. 2010; 37:217-245

4. Patel AL, Johnson TJ, Engstrom JL, et al. Impact of early human milk on sepsis and health care costs in very low birthweigh infants. J Perinatol. 2013; 33: 514-519.

5. Sisk PM, Lovelady CA, Dillard RG, Gruber KJ, O'Shea TM. Human milk consumption and full enteral feeding among infants who weigh <1250 grams. Pediatrics. 2008; 121: e128-e1533.

6. Meinzen-Derr J, Poindexter B, Wrage L, Morrow AL, Stoll B, Donovan EF. Role of human milk in extremely low birth weight infants' risk of necrotizing enterocolitis or death. $J$ Perinatol. 2009: 29: 57-62.

7. Furman L, Taylor G, Minich N, Hack M. The effect of maternal milk on neonatal morbidity of very low-birthweight infants. Arch Pediatr Adolesc Med. 2004: 157: 66-71. 
8. Johnson TJ, Patel AL, Jegier B, Engstrom JL, Meier J. The cost of morbidities in very low birth weight infants. J Pediatr. 2013; 162: 243-249.

9. Johnson TJ. The cost-effectiveness of human milk feedings as a strategy to reduce the risk of prematurity-specific morbidities in VLBW infants. Proceedings Experimental Biology Meeting; April 20-24, 2013 Boston, MA. (p. 61).

10. Infant and Young Child Feeding. World Health Organization website http://www.who.int/mediacentre/factsheets/fs342/en/. Updated February 2014. Accessed November 5, 2014

11. de Azevedo M, Mendes EN. Maintenance of lactation: a challenge for hospitalized premature infant's mothers. Rev Gaucha Enferm. 2008; 29(1): 68-75.

12. McGrath J. Invitation to develop targeted interventions to support continued direct breast-feeding in the NICU. J Perinat Neonatal Nurs. 2012; 26(4): 286-288.

Doi:10.1097/JPN.0b013e318272caea.

13. Pineda R. Direct breast-feeding in the neonatal intensive care unit: Is it important? J Perinatol. 2011; 31: 540-545.

Doi:10.1038/jp2010.205. 
14. Dowling D, Blatz MA, Graham G. Mothers' experiences expressing breast milk for their preterm infants: Does NICU design make a difference? Adv Neonatal Care. 2012; 12(4): 377-384.

15. LaSorte M. Replication as a verification technique in survey research: A paradigm. Sociol Q. 1972; 13: 218-227.

16. Finifter B. Replication and extension of social research through secondary analysis. Soc Science Info. 1975; 14: 119-153.

17. McCain GC. An evidence-based guideline for introducing oral feeding to healthy preterm infants. Neonatal Netw, 2003; 22(5): 45-50.

18. Maastrup R, Hansen BM, Kronborg H, et al. Breastfeeding progression in preterm infants is influenced by factors in infants, mothers and clinical practice: the results of a national cohort study with high breastfeeding initiation rates. PLoS One. 2014; 9(9):e108208. Doi:10.1371/journale.pone.0108208.

19. Hurst N, Engebretson J, Mahoney J. Providing mother's own milk in the context of the NICU: A paradoxical experience. $J$ Hum Lact. 2013; 29(3): 366-373.

20. Meier PP, Engstrom JL, Janes JE, Jegier BJ and Loera F. Breast pump suction patterns that mimic the human infant 
during breastfeeding: greater milk output in less time spent pumping for breast pump-dependent mothers with premature infants. J Perinatol. 2012; 32: 103-110. Doi: 10.1038/jp.2011.

21. Lucas R, Paquette RJ, Briere CE \& McGrath JM. Furthering our understanding of the needs of mothers who are pumping breast milk for infants in the NICU: An integrative review. Adv Neonatal Care. 2014; 14(4): 241-252.

22. Dieterich CM, Felice, JP, O'Sullivan, E, Rasmussen, KM. Breastfeeding and health outcomes for the mother-infant dyad. Pediatr Clin North Am. 2013; 60(1): 31-48.

23. Phung M, Grizzle V, Bethea J, et al. First feeding at breast - a simple change in significant improvement. Conference proceedings AWHONN 2014 Annual Education Meeting, June, 2014, Orlando, FL.

24. Mezzacappa, ES, Katkin, ES. Breastfeeding is associated with reduced perceived stress and negative mood in mothers. Health Psychology, 2002; 21(2): 187-193.

25. Guesnet, P, Alessandri, JM. Docosahexaenoic acid (DHA) and the developing central nervous system (CNS) - Implications for dietary recommendations. Biochimie, 2011; 93: 7-12. 
26. Li R, Fein SB, Chen J, Grummer-Strawn LM. Why mothers stop breastfeeding: mothers' self reported reasons for stopping during the first year. Pediatrics, 2008; 122(2): 9-76.

27. McFadden A, Toole G. Exploring womens views of breast feeding: a focus group study in an area with high levels of socioeconomic deprivation. Maternal \& Child Nutrition, 2006, 2: 156168.

28. Brown A. Maternal trait personality and breastfeeding duration: the importance of confidence and social support. Journal of Advanced Nursing, 2013; 70(3), 587-598. 